
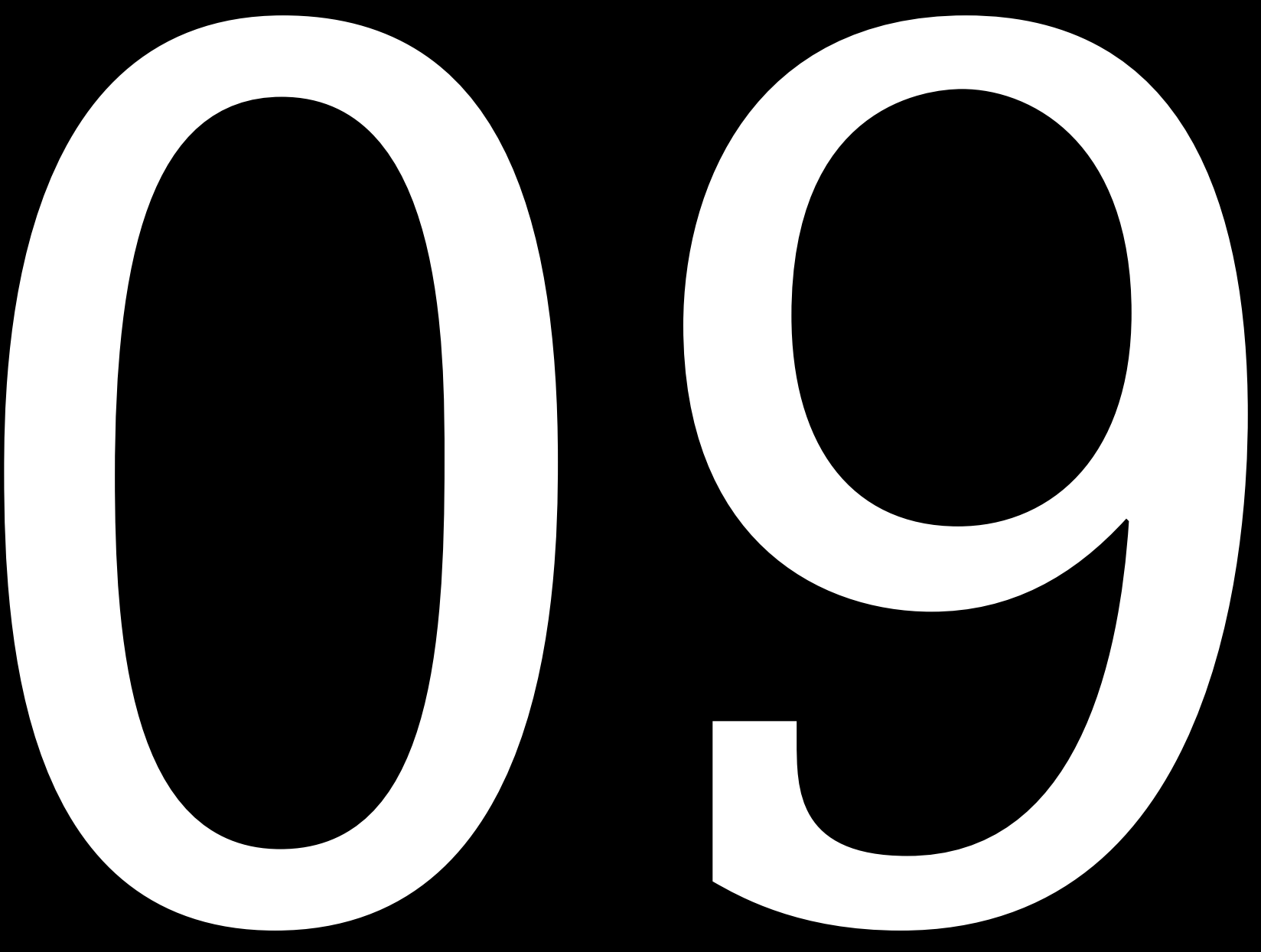

DOI: https://doi.org/10.14483/2422278X.16675 


\section{Más allá del escenario de la protesta social: los derechos humanos desde una perspectiva estudiantil}

Entrevista realizada por:

\section{Wilson Díaz Gamba ${ }^{1}$ Angely Katherine Torres Melo ${ }^{2}$}

Para citar: Díaz Gamba, W. \& Torres Melo, A. K. (2020). Más allá del escenario de la protesta social: los derechos humanos desde una perspectiva estudiantil. Ciudad Paz-ando, 12(2), 111-117. doi: https://doi.org/10.14483/2422278X.16675

Privar a las personas de sus derechos humanos es poner en tela de juicio su propia humanidad. Nelson Mandela

Una persona antes de ser mujer u hombre, ingeniera(o), licenciada(o) o administrador(a), es un ser humano. Dicha condición no es una característica innata, sino una que requiere confirmación por medio de la interacción y la comunión con los demás, lo que establece que la humanidad tiene un componente natural y otro artificial (Savater, 2002). El primero alude al proceso biológico con el que nacen las personas, mientras que el segundo es el que se da en la formación e interacción dentro de la sociedad.

El hombre es malo por naturaleza, diría (Hobbes, 2008) o en términos de Freud (1995) personas poseen una pulsión destructiva inherente a su ser, que los lleva a expresar toda la violencia arcaica que poseen. Para contener dicha pulsión, se requiere de un proceso de sociabilización en el cual la persona aprende a contener sus instintos, al interiorizar las normas y los valores de la cultura en la que vive. Por lo tanto, puede afirmarse que no hay nada más útil a la humanidad que la humanidad,

1 Director del Instituto para la Pedagogía, la Paz y el Conflicto Urbano (Ipazud) de la Universidad Distrital Francisco José de Caldas y de la Revista Científica Ciudad Paz-ando, editada por el mismo instituto. Magíster en Investigación Social Interdisciplinaria, especialista en Desarrollo Humano y licenciado en Ciencias Sociales de la Universidad Distrital Francisco José de Caldas. Además, es Psicólogo de la Institución Universitaria Politécnico Grancolombiano y docente titular del área de humanidades de la Facultad de Ingeniería de la Universidad Distrital Francisco José de Caldas

2 Licenciada en Ciencias Sociales, maestrante en Educación con énfasis en Comunicación Intercultural, Etnoeducación y Diversidad Cultural de la Universidad Distrital Francisco José de Caldas. Actualmente es investigadora principal de la línea de investigación Derechos Humanos y Equidad de Género del Instituto para la Pedagogía, la Paz y el Conflicto Urbano de la Universidad Distrital Francisco José de Caldas. 
como lo diría Spinoza citado por Damasio (2005) y que la humanización es un proceso recíproco que se construye en relación con los demás.

Este proceso de humanización, que en un momento fue potestad del clan y la familia, en la modernidad fue un rol que se le adjudicó a la escuela como principio primordial en la socialización. Hoy en día pareciera que esta labor culmina en los procesos básicos de formación, y que al ingresar a la educación superior no es necesario seguir reflexionando sobre la humanización, ya que lo transcendental en este nivel de la educación pareciera ser el de capacitar para la producción y la economía, olvidando que las personas nunca dejan de aprender, que la educación no sólo debe preparar para el trabajo sino para el ejercicio de la ciudadanía y la convivencia, debe formar para vivir y mantener la democracia, sobre todo cuando es en la universidad que los jóvenes se asumen como ciudadanos.

En los últimos años, y dado el aumento de la protesta social en nuestro país, en las universidades públicas, principalmente, hemos visto a los jóvenes reaccionar de maneras propositivas frente al uso desmedido de la fuerza pública en estos escenarios, ellas(os) se han organizado para defender la vida y la integridad de las(os) estudiantes que participan en las movilizaciones, lo que ha configu-

rado nuevas formas de pensar el reconocimiento del otro(a), ha construido tejido social desde el sentido de lo humano como camino a la resolución de conflictos y, sobre todo, partiendo de principios inherentes a todas(os), los derechos humanos. De acuerdo con lo anterior, desde el Instituto de Paz de la Universidad Distrital Francisco José de Caldas hemos querido conocer sobre los fines, propósitos y acciones que estas(os) jóvenes realizan al interior del alma mater.

En este sentido, se convocó a los integrantes del Comité Estudiantil de Derechos Humanos de la Universidad Distrital, y se generó un grupo de discusión que permitió abordar las siguientes preguntas formuladas por el Ipazud, a las cuales cada integrante respondió dando una visión general consensuada por la colectividad que conforman.

\section{1. ¿Qué es el Comité estudiantil de DDHH y cómo se originó?}

El comité es una expresión organizativa del movimiento estudiantil que se da a la tarea de defender los derechos humanos, especialmente de los estudiantes, en los escenarios de protesta y movilización social. Este surge como respuesta a las constantes violaciones de Derechos Humanos por parte del Escuadrón Móvil Anti-Disturbios (Esmad) para reprimir las movilizaciones de los paros estudiantiles.

Este comité se origina en uno de los Encuentros Nacionales de Estudiantes de Educación Superior (Enees), donde se hizo el llamado a cada universidad para conformar su comité durante el paro nacional universitario del 2018. En un principio, era un espacio de trabajo político de la Unión Nacional de Estudiantes de Educación Superior (Unees); sin embargo, con el paso del tiempo terminó siendo independiente de dicha plataforma. De allí que el Comité sea un lugar de base amplio para la articulación de diferentes estudiantes, sin importar su postura política, ideológica o social, con el fin de concretar un proyecto de defensa de los derechos humanos, encaminado a blindar los ejercicios de movilización estudiantil y velar por condiciones dignas del estudiantado y la garantía de sus derechos en la vida universitaria. Cabe resaltar que desde el momento en que el comité se consolidó, bajaron notablemente las estadísticas de estudiantes de la Universidad Distrital que eran retenidos arbitrariamente.

\section{Hablemos de la organización interna, ¿cómo se da la conformación del Comité y cómo se llega a ser parte de él?}

El Comité está integrado por estudiantes de las sedes de la Universidad Distrital y entiende las diferentes dinámicas en sus formas de movilización social y las condiciones

"Entendemos los
Derechos Huma-
nos como princi-
pios, inherentes a
todos y todas, que
constituyen con-
diciones para la
vida digna de las

particulares de la vida universitaria. En este sentido, cada sede requiere formas específicas de defensa de los Derechos Humanos y por esta razón el trabajo del comité inicia desde lo local y se consolida como una articulación amplia que abarca la labor de defensa en toda la universidad. Actualmente, tiene una mayor participación de las sedes Macarena, Vivero, Tecnológica e Ingeniería, y dentro de él coexisten varias expresiones políticas, organizativas e individualidades que nutren nuestras acciones, tanto en campo, como en lo que llamamos trabajo de oficina.

Cualquier estudiante puede ser defensor(a) de Derechos Humanos e integrante del Comité, la única condición es estar activo dentro de la universidad. Además, es necesario estar interesado y comprometido en esta labor voluntaria, acercarse a los espacios y procesos de base generados en las sedes para recibir una formación, en teoría y en campo, que los mismos integrantes enseñan y en la que las personas adquieren nociones básicas de Derechos Humanos, su ejercicio y defensa en escenarios de protesta. Estas jornadas de formación se proyectan una vez al año mediante una convocatoria abierta a todos los estudiantes que desean participar en los diferentes ciclos. Una vez las personas nuevas y el comité terminen el proceso, se implementa el trabajo práctico, teniendo en cuenta que siempre los nuevos(as) integrantes estarán acompañados(as) de quienes tienen más experiencia. 


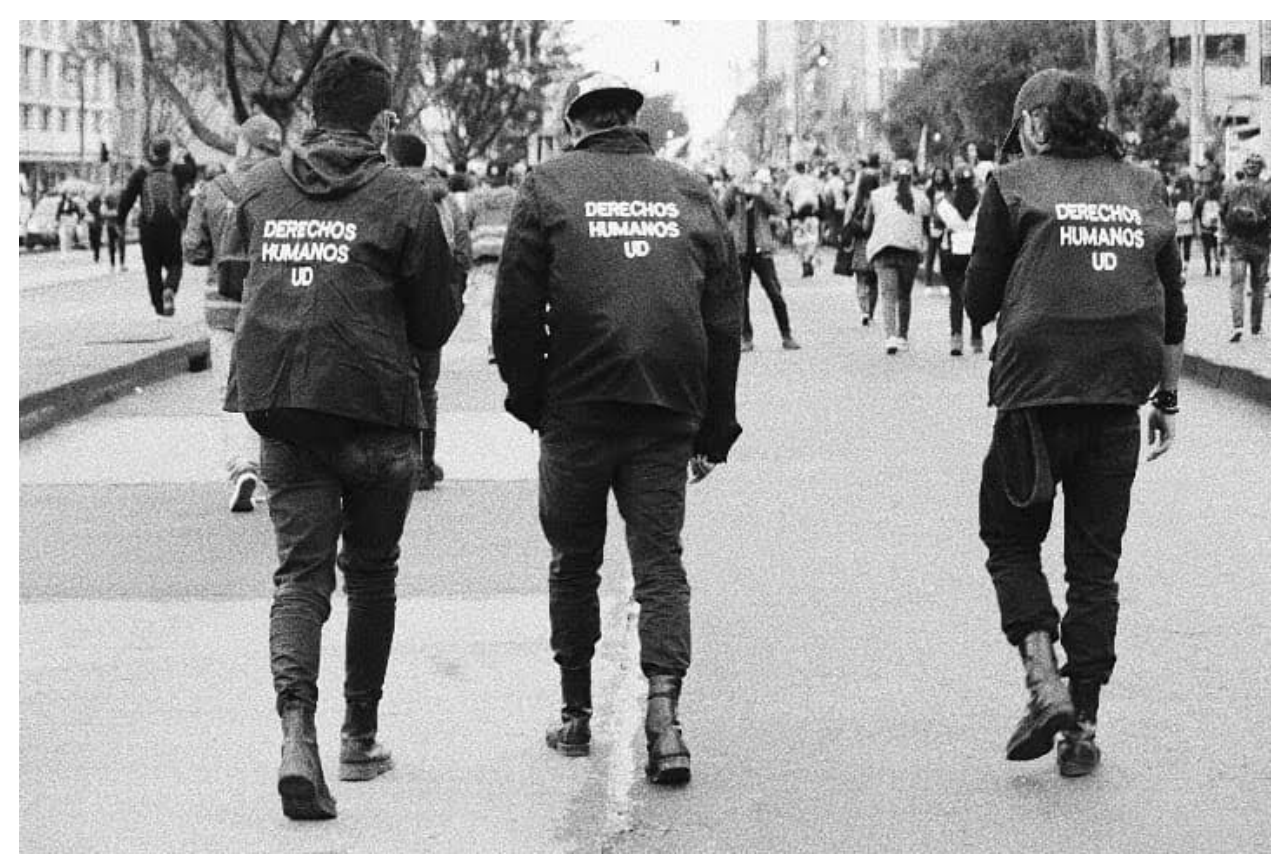

3. ¿Cómo se entiende el concepto de DerechosHumanosdesdelaperspectiva de los integrantes del Comité?

Entendemos los derechos humanos como principios inherentes a todos y todas, que constituyen condiciones para la vida digna de las personas. Aunque su origen sea liberal, éstos son victorias de los pueblos que reivindican su existencia y, en muchas ocasiones, nuestra única herramienta contra el capitalismo exacerbado. Dentro de la vida universitaria, estos son la bandera de la protección de la comunidad estudiantil frente a abusos, vulneraciones y acciones que van en contra de la integridad fisica y de la misma dignidad humana. Por tanto, son un aspecto transversal que debe estar presente en las diversas actividades y escenarios de la universidad, así como en todos los ámbitos de la vida social.

En otras palabras, los derechos humanos son todas las acciones que llevamos a cabo desde salir a una marcha hasta ponerse un chaleco, pues nuestra prioridad está en los escenarios de protesta, partiendo de dos derechos fundamentales: el derecho a la vida y el derecho a la protesta. Comprendemos que los derechos son universales, por lo cual no restringimos nuestra atención a ningún tipo de manifestantes -sin importar su forma de manifestarse-, ya que entendemos que el Estado es el único que tiene la obligación de garanti-

“...entre los aspectos más significativos, destacamos el reconocimiento y agradecimiento que los y las estudiantes manifiestan cada vez que actuamos en defensa de su integridad.". zar los derechos de las personas. Por lo anterior, cualquier vulneración de derechos humanos es responsabilidad de este, ya sea por acción o por omisión, sobre todo teniendo en cuenta que es en los escenarios de protesta donde hemos evidenciado las constantes vulneraciones a la integridad de manifestantes y no manifestantes por parte del Esmad. En este horizonte se desarrolla el componente de denuncia de estas acciones por parte del comité.

\section{Además de la defensa de los Derechos Humanos en el marco de las situaciones presentadas en la protesta social, ¿qué otros escenarios en la cotidianidad universitaria reflejan situaciones de vulneración en los que el comité proceda en su accionar?}

Aunque el enfoque del comité enfatiza en la defensa del derecho a la vida y a la protesta, cada integrante tiene formación integral en derechos humanos, por lo tanto, también actuamos en su defensa en la cotidianidad universitaria. En este escenario, los campos de disputa amplios incluyen temas de autonomía universitaria, bienestar universitario (apoyo alimentario), infraestructura (caída de techos), estigmatización y persecución, además de las violencias basadas en género, en las cuales se han denunciado diferentes situaciones que evidencian la existencia de un sistema patriarcal de base y una cultura machista, tema en el cual las compañeras del comité de género han profundizado aún más, pero que también entendemos como una lucha por la garantía de los derechos humanos.

En este sentido, podemos decir que el Comité se ha pronunciado al denunciar, rechazar y visibilizar acciones de vulneración a los derechos humanos que, desde 
diferentes ámbitos y actores, se llevan a cabo en detrimento de condiciones de vida digna para la comunidad universitaria.

\section{Dentro de su ejercicio como defensor(a) de los Derechos Humanos al interior de la Universidad, ¿cuáles han sido las experiencias más significativas dentro de su proceso?}

Las experiencias más significativas están relacionadas con varios ámbitos. Una de ellas ha sido el proceso de capacitación, ya que a partir de la formación, cada defensor(a) logra una mayor comprensión de las dinámicas sociales, al mismo tiempo que reflexiona, problematiza y concientiza acerca de los riesgos que tiene defender los derechos humanos en contextos como el nuestro, gravemente afectados por una historia de guerra e injusticias. Sin embargo, es motivo de alegría ver cómo los compañeros y compañeras participan más en el debate de la defensa de los derechos, ya que es a partir de la participación que se logra avanzar en este trabajo. En este orden de ideas, se destacan dos procesos: el realizado en la Facultad de Ingeniería durante el 2019, y el llevado a cabo en la Macarena este año, pues además de enriquecer el bagaje en la labor, propiciaron la participación en la consolidación del valioso proceso organizativo del Comité Estudiantil de Derechos Humanos.

Otra de las experiencias atañe a las diferentes oportunidades en las que se realiza la verificación de derechos humanos in situ durante escenarios de protesta en la modalidad de acción directa en dife-

"...siempre propen-

demos por dialogar con la fuerza pública, entidades gubernamentales $y$ veedoras, para que se blinde el ejercicio legítimo de la protesta pacífica como acto democrático.". las estudiantes manifiestan cada vez que actuamos en defensa de su integridad.

\section{Hablando del momento coyuntural por el que atraviesa el país, en la difícil situación de la implementación del acuerdo de paz y la reconciliación nacional, ¿qué lugar ocupa el diálogo en el desarrollo de las acciones como comité en campo?}

La labor de defensa de los derechos humanos realizada por el Comité en los escenarios de protesta social se nutre del diálogo como principal instrumento para evitar la confrontación y la manifestación de formas de violencia que atenten contra los derechos del estudiantado. Como comité, creemos que generar canales de interlocución y soluciones dialogadas a los conflictos es una vía legal y política efectiva para evitar la vulneración de los derechos humanos; por lo tanto, en campo siempre propendemos por dialogar con la fuerza pública, entidades gubernamentales y veedoras para que se blinde el ejercicio legítimo de la protesta pacífica como acto democrático. Asimismo, enfatizamos en la importancia de mantener un canal de enlace permanente y una comunicación abierta con los estudiantes. La comprensión de situaciones y realidades permiten la eficacia de nuestra labor.

Pensándonos este escenario dentro del contexto nacional, la defensa de los derechos humanos y las garantías plenas a la protesta son dos condiciones imprescindibles para materializar la paz social y superar la violencia sociopolítica aún marcada en el país. En este sentido, entendemos que el diálogo es fundamental en los procesos de interlocución; sin embargo, en muchas ocasiones se ve relegado por las mismas autoridades policiales, pues no brindan información sobre detenidos o procedimientos, lo que hace cada vez más difícil la efectividad del proceso. Así las cosas, consideramos pertinente retomar una definición de paz, no como cese de la violencia directa, sino considerando los múltiples factores sociales, políticos y económicos por resolver.

De esta manera, comprendemos que es difícil hablar de paz cuando el Estado constantemente hace uso de la fuerza para agredir a la población civil que demuestra su inconformismo con problemas estructurales, más cuando se difunden discursos de odio que justifican la violencia estatal y de otros actores en conflicto. Esto podría llevar a interpretar que el diálogo sigue teniendo un lugar utópico, muy difícil de alcanzar, ya que la población no cree en su efectividad y se necesita del reconocimiento de otro(a) como interlocutor(a) válido(a), además del compromiso del Estado para que este sea viable. 


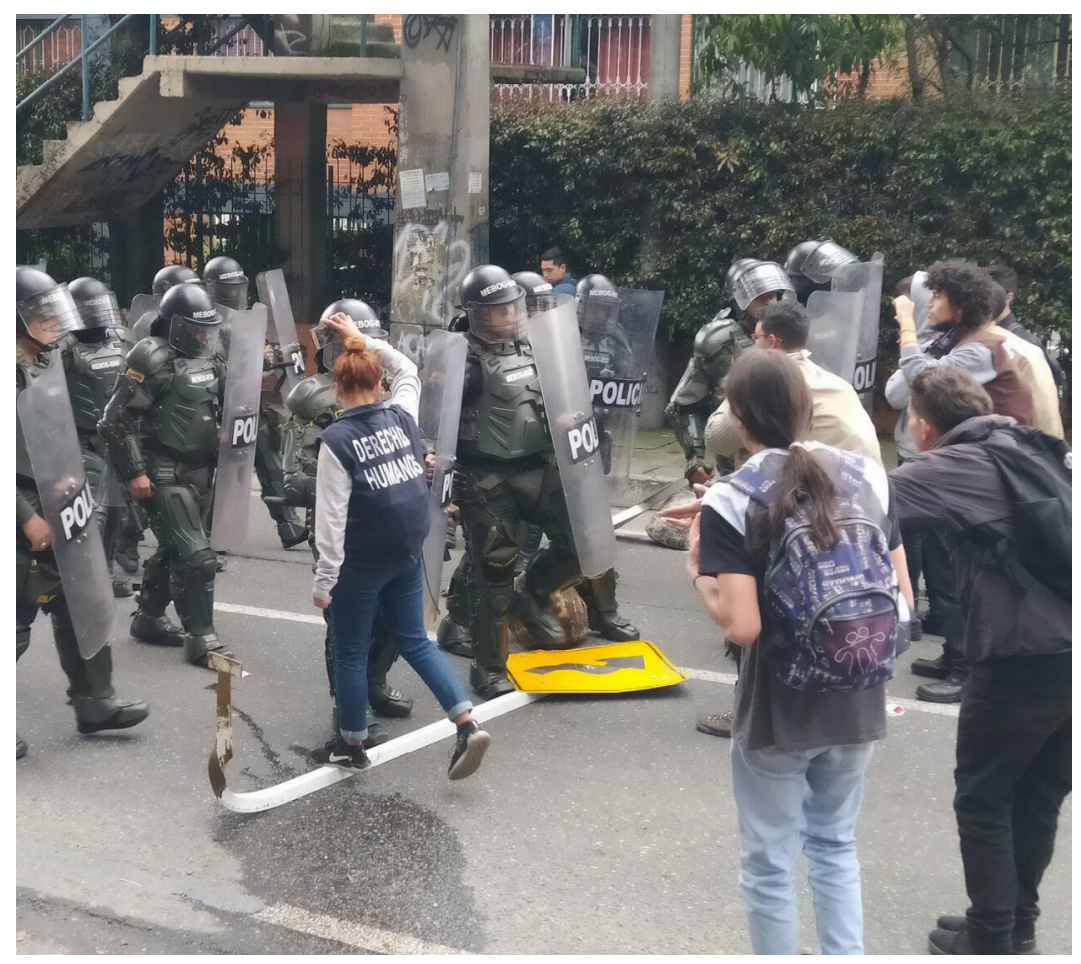

\section{7. ¿Qué experiencias relevantes se han dado en el trabajo en campo ejerciendo como defensor de Derechos Humanos?}

Las movilizaciones estudiantiles realizadas desde el mes de septiembre del 2019 fueron una experiencia significativa en la labor que hemos ejercido desde el Comité de Derechos Humanos. La coyuntura de protestas en Bogotá durante la semana del 23 al 27 de septiembre, motivadas por el rechazo e indignación hacia el escándalo de corrupción surgido en la Universidad Distrital, fue una gran y ardua experiencia que fortaleció el trabajo colectivo del comité. Asimismo, las movilizaciones del 24 y 31 de octubre en Bogotá marcaron una experiencia importante en términos de unión y coordinación, en tanto que la labor se empezó a manejar de forma interuniversitaria, lo que fortaleció el trabajo desde la Red Universitaria de Derechos Humanos, de la cual participamos activamente. Una experiencia única se dio durante la coyuntura del Paro Nacional, a finales del año 2019, en la cual nuestra labor se hizo más solidaria al respaldar las manifestaciones en los barrios y localidades, y al lograr la articulación con organizaciones sociales, entendiendo que la protesta es un derecho fundamental en los diferentes escenarios de movilización y reivindicación social.

Existen gran cantidad de sucesos y situaciones dentro del trabajo en campo que llevan a reflexionar sobre los discursos que se manejan dentro de la policía, la estigmatización constante hacia el movimiento estudiantil y la justificación del accionar violento sin dar respuesta a las necesidades, demandas y reivindicaciones de los(as) estudiantes, además de la intencionalidad de evitar nuestra labor de defensa de los derechos humanos por parte de los cuerpos policiales. Conocer los cientos de casos de desapariciones, violencias basadas en género y las agresiones físicas de los CTP moviliza el pensamiento y la acción desde el sentido de lo humano. Es importante traer a colación el recuerdo de una joven que en medio de una movilización frente al proceder del Esmad entra en pánico y queda paralizada, sola, contra una pared, cuando la movilización ya se estaba dispersando, pero la intervención de los defensores(as) logra salvarla del ataque del escuadrón. Lo anterior demuestra la importancia de nuestra labor, ya que un accionar efectivo y a tiempo logra evitar vulneraciones a los derechos de manifestantes y no manifestantes.

\section{8. ¿Cuál debería ser el rol de la fuerza pública en los escenarios de protesta social?}

En relación con la protesta social, la fuerza pública debe actuar como un cuerpo cívico que propende por la garantía de los derechos y libertades fundamentales de los ciudadanos, y que defiende la convivencia y la paz social. Esta es su función constitucional y legal. La fuerza pública tiene un papel encaminado a garantizar la protesta social pacífica en su sentido pleno, y debe respetar las normatividades y protocolos ya establecidos. Nunca puede llevar a cabo acciones encaminadas a la estigmatización y represión de los manifestantes, independientemente de las motivaciones del ejercicio de protesta. Cuando hay acciones violentas en el marco de las movilizaciones sociales, la fuerza pública contiene los conatos de disturbio, 
empleando de forma proporcional la fuerza legítima que le confiere el Estado, mas no puede hacer un uso letal de la fuerza, como ya lo hemos evidenciado en múltiples ocasiones.

En este sentido, el comité reconoce que, en la actualidad, en términos prácticos el rol de la fuerza pública es agredir a quienes están inconformes con las problemáticas sociales y lo manifiestan, en vez de ser garante del bienestar externo de la movilización, se manifiesta como represiva y vulneradora de derechos, como lo hemos evidenciado en las movilizaciones. Así las cosas, no debería estar en los espacios de manifestación, pues ¿qué necesidad hay de tener un escuadrón de policía al lado de una movilización pacífica? Consideramos que esto sólo promueve la estigmatización a esta institución. De igual modo, es necesario llevar a cabo los procesos sancionatorios y punitivos pertinentes por los hechos de violación a los derechos cometidos por la fuerza pública.

\section{9. ¿Es la Universidad un escenario de violación de Derechos Humanos?}

Partiendo de la existencia de una violencia estructural, entendemos que en los diferentes ámbitos de la vida social se manifiestan todo tipo de violaciones a los derechos humanos, no sólo de primera generación, sino también de segunda y tercera. En este sentido, reconocemos que en la cotidianidad universitaria se evidencian múltiples vulneraciones a los derechos humanos, como el reflejo de un sistema macro. Esto se evidencia en la falta de inversión y de presupuesto considerable para la educación, materializada en las precariedades de infraestructura e impulso a la investigación, por dar un par de ejemplos. A esto se suman los actos de corrupción, que roban los recursos públicos destinados para estos fines misionales. Existe una grave transgresión a los derechos hacia la comunidad universitaria y la ciudadanía en general.

Cuando no se brindan condiciones de bienestar, flexibilidad académica, apoyo psicosocial y socioeconómico a estudiantes, y atención efectiva a casos de violencias basadas en género, hay una sistematicidad en la vulneración de los derechos de la comunidad universitaria. En definitiva, desde múltiples aspectos, la universidad puede ser un escenario potencial en el que se siguen violentando derechos y libertades, más aún cuando hay una ausencia de políticas, procedimientos y accionares que permitan la no reproducción de violencias y estigmatizaciones.

Asimismo, el derecho a la participación es uno de los más afectados, ya que se restringen los espacios de discusión y de decisión, como asambleas, donde, en muchas ocasiones, profesores, estudiantes o trabajadores reproducen estereotipos, representaciones e imaginarios de estigmatización hacia las diferentes formas de pensar y ser dentro de la universidad, lo que genera un ambiente de tensión que promueve la vulneración de otros derechos. Cabe anotar aquí que los problemas estructurales que producen todo tipo de amenazas a los derechos están atravesados por la violencia cultural que se materializa en las acciones cotidianas de cada uno(a); por eso, una de las propuestas frente a estas problemáticas es trabajar en el reconocimiento de la pluralidad de existencias y realidades, además de impulsar procesos formativos y pedagógicos que promuevan la discusión y sensibilización frente a dichos escenarios.

\section{0. ¿Por qué debe prohibirse el ingreso de la fuerza pública a los campos universitarios en los eventos de protesta y bloqueos alrededor de los centros educativos?}

La violencia como respuesta a la violencia nunca será una solución que mitigue la problemática; sino todo lo contrario, va a generar radicalizaciones y respuestas re“...una de las pro- iterativas por la misma vía. Es evidente puestas frente a estas problemáticas es trabajar en el reconocimiento de la pluralidad de existencias y realidades, además de impulsar procesos formativos y pedagógicos que promuevan la discusión y sensibilización frente a dichos escenarios.". de manera irregular de forma sistemáti$\mathrm{ca}$, por lo que permitir su ingreso a los campus universitarios pondría en peligro a las personas que no están realizando ninguna acción violenta, al exponerlas a abusos y arbitrariedades. Si se permite el ingreso, se vulnera el derecho a la autonomía universitaria, y, además, se violenta el constante flujo de ideas y expresiones del movimiento estudiantil. Así las cosas, ¿quién se haría responsable de lo que pase dentro del campus en el momento del ingreso de la fuerza pública? ¿Qué pasaría si aparece alguien muerto o si un(a) compañero(a) es abusado(a)? Frente a esto, es necesario señalar que la fuerza pública nos ha demostrado constantemente cómo abusa de su autoridad y hace uso excesivo de la fuerza, por lo cual es un peligro contra la comunidad universitaria el dejarles entrar.

El uso de las universidades públicas como escudos sí es una problemática, pero a esto no se debe responder con el uso desmedido de la fuerza para solucionar el problema de la acción violenta generada en las universidades. Sería más adecuado atender las demandas que se piden y las problemáticas que se denuncian por medio de las manifestaciones, lo que señala que solucionando estructuralmente las exigencias, se superan las expresiones 


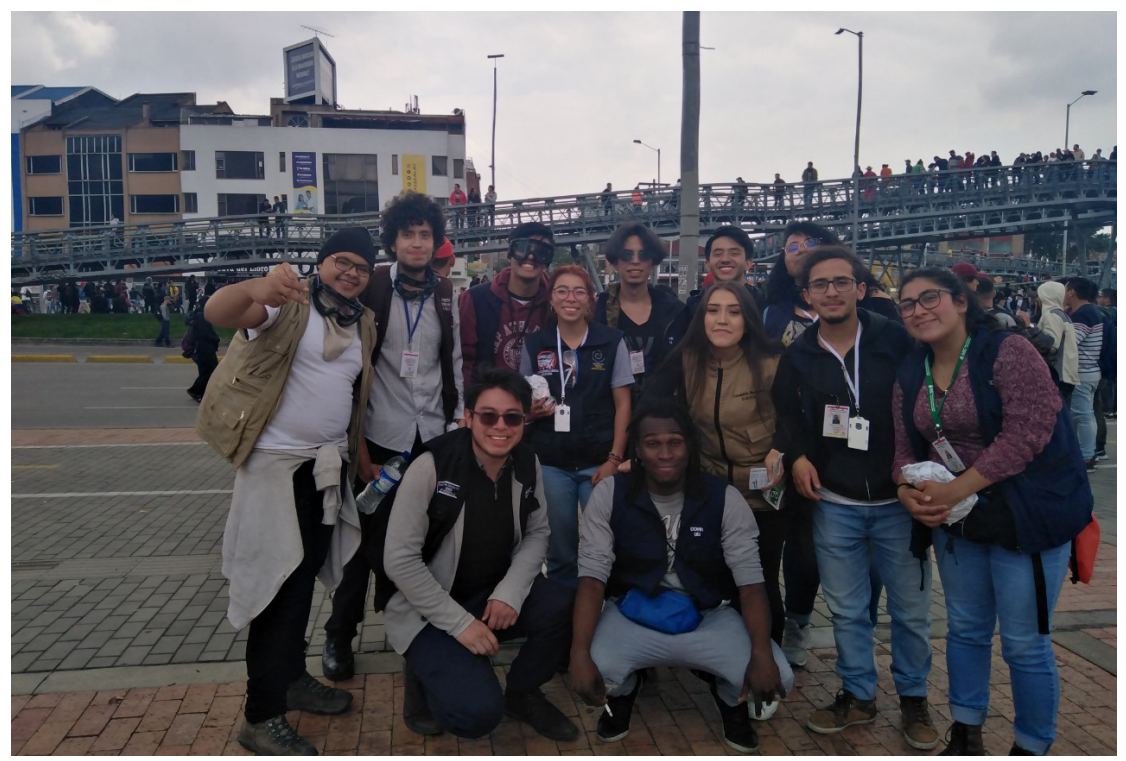

violentas y se avanza hacia formas democráticas más claras y efectivas. Sin embargo, frente al punto de la acción violenta, el comité se encuentra en constante discusión, debido a la pluralidad de perspectivas, su naturaleza y composición.

\section{Una cultura de la defensa de los Derechos Humanos: cerrando la brecha entre teoría y práctica}

La discusión suscitada con los estudiantes plantea diferentes campos y caminos de abordaje, teniendo en cuenta la transversalidad del concepto y ejercicio de los derechos humanos. Cabe resaltar la importancia que tiene para la institucionalidad colombiana, distrital y para la ciudadanía en general, el hecho de que las(os) jóvenes se apropien del discurso por la vida y la dignidad humana, y hagan de él la carta de navegación para afrontar los diferentes escenarios de su vida cotidiana. Así las cosas, y como bien lo han resaltado las(os) integrantes del comité, es necesario pensarse los ámbitos de formación, sensibilización, denuncia y atención inmediata de las situaciones que vulneran o amenazan la vida, así como la integridad y todos los derechos en su conjunto, sean de primera, segunda o tercera generación.

Esto lleva a cuestionarse sobre el rol y el aporte que generamos todas(os) desde los diferentes lugares y campos que ejercemos como integrantes de la sociedad y, especialmente, de la universidad, entendiendo esta, no sólo como el escenario que refleja todas aquellas dinámicas y problemáticas del país y el mundo, sino como el eje generador de propuestas y ejercicios práxicos de transformación de realidades. En este sentido, hablar de derechos humanos implica partir del reconocimiento de la pluralidad de existencias, y, una vez hecho esto, debemos comprender que su defensa y ejercicio depende del trabajo articulado en favor del mismo objetivo, de tejer las condiciones colectivas para el respeto y la dignidad. Pensar políticas, procedimientos y accionares desde lo institucional, que enmarquen los objetivos, necesita de un trabajo participativo, vinculante y, sobre todo, que reconozca las realidades de los sujetos, para cerrar la brecha entre teoría y práctica, para que el concepto no se quede en el escritorio y pueda ser realmente efectivo en su vivencia cotidiana.

Las(os) jóvenes que lideran este tipo de iniciativas, de alguna manera están mostrándole a la ciudadanía la posibilidad de construir propuestas creativas, diferentes a las que han aprendido a través de una historia cruenta de guerra e injusticias, pues enarbolar la bandera por la defensa de los derechos humanos responde a la necesidad de humanidad en los escenarios que atraviesan. Este escenario se convierte en una oportunidad precisa para que estudiantes, docentes, trabajadores y directivos de la universidad trabajen colaborativamente en la construcción de una cultura que promueva la defensa de los derechos humanos, claramente atravesada por un enfoque de género, en la que todas(os) - a pesar de nuestras diferencias políticas, ideológicas, generacionales, sexuales y/o culturales- adoptemos los principios de respeto por la vida, la dignidad y la libertad desde el sentido de lo humano.

\section{Referencias}

Damasio, A. (2005). En busca de Spinoza. Neurobiología de la emoción y los sentimientos. Crítica.

Freud, S. (1995). Los origenes del psicoanálisis. Alianza.

Hobbes, T. (2008). Tratado sobre el ciudadano. Universidad Nacional de Educación a Distancia.

Savater, F. (2002). Ética y ciudadanía. Montesinos.

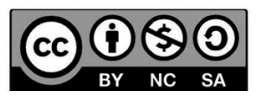

Ciudad Paz-ando, Bogotá. Julio - Diciembre 2019. Vol 12.2, 111-117 